PROCEEDINGS OF THE

AMERICAN MATHEMATICAL SOCIETY

Volume 127, Number 8, Pages 2375-2384

S 0002-9939(99)04806-6

Article electronically published on April 8, 1999

\title{
CONSTRUCTIVE DECOMPOSITION OF FUNCTIONS OF FINITE CENTRAL MEAN OSCILLATION
}

\author{
J. D. LAKEY
}

(Communicated by J. Marshall Ash)

\begin{abstract}
The space CMO of functions of finite central mean oscillation is an analogue of $\mathrm{BMO}$ where the condition that the sharp maximal function is bounded is replaced by the convergence of the sharp function at the origin. In this paper it is shown that each element of $\mathrm{CMO}$ is a singular integral image of an element of the Beurling space $B^{2}$ of functions whose Hardy-Littlewood maximal function converges at zero. This result is an analogue of Uchiyama's constructive decomposition of $\mathrm{BMO}$ in terms of singular integral images of bounded functions. The argument shows, in fact, that to each element of $\mathrm{CMO}$ one can construct a vector Calderón-Zygmund operator that maps that element into the proper subspace $B^{2}$.
\end{abstract}

\section{INTRODUCTION}

A function $f$, defined modulo constants on $\mathbb{R}^{n}$, is said to have finite central mean oscillation provided the $L^{2}$ normalized sharp function of $f$ converges at $x=0$. That is,

$$
M_{2}^{\sharp} f(0)=\left(\sup _{R>0} \frac{1}{R^{n}} \int_{|x|<R}\left|f(x)-f_{R}\right|^{2} d x\right)^{1 / 2}<\infty,
$$

where $f_{R}$ is the average of $f$ over $B(0 ; R)$. The space of all such functions is denoted by $\mathrm{CMO}\left(\mathbb{R}^{n}\right)$ and $M_{2}^{\sharp} f(0)$ defines a norm on this space.

The space CMO bears a simple relationship with BMO, the space of functions of bounded mean oscillation: $g \in$ BMO precisely when $g$ and all of its translates belong to CMO uniformly a.e., that is, when the sharp function of $g$ belongs to $L^{\infty}$. As demonstrated in work of Chen and Lau [3] who first defined CMO, of Garcia-Cuerva [4] (they define inhomogeneous versions, restricting to $R \geq 1$ in (1.1)), and of Chao, Gilbert, and Tomas [1], many precise analogies exist between $\mathrm{CMO}$ and $\mathrm{BMO}$ from the point of view of real Hardy spaces. In fact, the purpose of the present paper is to draw a further analogy: to develop a version of Uchiyama's constructive decomposition of $\mathrm{BMO}$ in terms of sums of images of bounded functions under certain singular integrals, in the context of CMO. Facts about CMO are not always simpler versions of facts about BMO. For example, there is no JohnNirenberg lemma for CMO. That lemma implies that the $L^{\infty}$ norms of $M_{p}^{\sharp} g(x)$,

Received by the editors January 31, 1997 and, in revised form, November 1, 1997.

1991 Mathematics Subject Classification. Primary 42B20, 42B30.

Key words and phrases. Hardy space, Beurling space, singular integral, wavelets.

The author was supported in part by NMSU grant \# RC96018. 
where 2 is replaced by some other $p, 1 \leq p<\infty$ in (1.1), define equivalent norms on BMO. But as Chen and Lau showed, the condition $M_{p}^{\sharp} g(0)<\infty, p \neq 2$, defines a space $\mathrm{CMO}^{p}$ different from CMO. In the sequel, by CMO we will always mean $\mathrm{CMO}^{2}$.

$\mathrm{CMO}\left(\mathbb{R}^{n}\right)$ is the dual of the Beurling-Hardy space $H A^{1}\left(\mathbb{R}^{n}\right)$. This space and the Beurling algebra $A\left(\mathbb{R}^{n}\right)$ upon which it is built are given by:

\section{Definition 1.2.}

$$
\begin{aligned}
A\left(\mathbb{R}^{n}\right) & =\left\{f: \mathbb{R}^{n} \rightarrow \mathbb{C}:\|f\|_{A}=\sum_{j \in \mathbb{Z}} 2^{n j / 2}\left\|f \chi_{\left\{2^{j} \leq|x| \leq 2^{j+1}\right\}}\right\|_{L^{2}}<\infty\right\} ; \\
H A^{1}\left(\mathbb{R}^{n}\right) & =\left\{f: S(f)(x)=\left(\iint_{|x-y| \leq t}|\nabla f(y, t)|^{2} d y d t\right)^{1 / 2} \in A\left(\mathbb{R}^{n}\right)\right\} .
\end{aligned}
$$

The relationship between $H A^{1}\left(\mathbb{R}^{n}\right)$ and $A\left(\mathbb{R}^{n}\right)$ is the same as that between the real Hardy space $H^{1}\left(\mathbb{R}^{n}\right)$ and $L^{1}\left(\mathbb{R}^{n}\right)$. The dual of $A\left(\mathbb{R}^{n}\right)$ is the Beurling space

$$
B^{2}\left(\mathbb{R}^{n}\right)=\left\{h: M_{2} h(0)=\left(\sup _{R>0} \frac{1}{R^{n}} \int_{|x|<R}|h(x)|^{2}\right)^{1 / 2}<\infty\right\},
$$

and the $H A^{1} \leftrightarrow$ CMO duality is then analogous to the $H^{1} \leftrightarrow$ BMO duality. In the latter case, $f \in \mathrm{BMO}$ is the same as $M_{2}^{\sharp} f$ belonging to the dual of $L^{1}$. In the former case, $g \in \mathrm{CMO}$ is the same as $M_{2}^{\sharp} g$ belonging to the dual of $A$, a corollary of the fact that $M_{2}$ is bounded on $B^{2}$ just as it is on $L^{\infty}$ (cf. [3]).

Among the real-variable analogies between these pairs of spaces is the fact that standard singular integrals map $H A^{1}$ into $A$ just as they map $H^{1}$ into $L^{1}$ (cf. $[4])$; and just as the identity $I$ and Riesz transforms $R_{j}: f \mapsto c_{n}$ p.v. $f * \frac{x_{j}}{|x|^{n+1}}$ together characterize $H^{1}$ and BMO in the sense that $f \in H^{1}\left(\mathbb{R}^{n}\right)$ if and only if $f, R_{1} f, \ldots, R_{n} f \in L^{1}\left(\mathbb{R}^{n}\right)$, Garcia-Cuerva showed that the same holds when $H^{1}$ and $L^{1}$ are replaced by $H A^{1}$ and $A$. A consequence of our present work is a constructive version of this Fefferman-Stein decomposition for CMO: $g \in \mathrm{CMO}\left(\mathbb{R}^{n}\right)$ if and only if one can construct $g=h_{0}-\sum_{j=1}^{n} R_{j} h_{j}$ where $h_{0}, h_{1}, \ldots, h_{n} \in B^{2}\left(\mathbb{R}^{n}\right)$.

The conditions defining $B^{2}$ and CMO are simpler than those defining $L^{\infty}$ and BMO because they only need to be checked at the origin - not at every point. In this sense, a constructive version of Uchiyama's result should be simpler for these spaces. Yet there are still good reasons for carrying out that program in the present setting. First, the constructive decomposition argument that we propose for CMO has substantially new features; for example, there is no John-Nirenberg lemma to use. The argument is simpler from a computational standpoint than Uchiyama's. This could potentially lead to a simpler version of the constructive decomposition of BMO and more tractable attacks on problems in Hardy space theory such as existence of boundary values of harmonic functions that use Uchiyama's techniques, e.g., [7]. The second reason is that we can say more about the functions $h_{0}, h_{1}, \ldots, h_{n}$ in the Fefferman-Stein decomposition this way. In particular, if $g \in \mathrm{CMO}$, then there are Calderón-Zygmund operators (CZO's) $L_{0}, L_{1}, \ldots, L_{n}$ such that $h_{j}=L_{j} g$ and such that $L_{0}-\sum_{j=1}^{n} R_{j} L_{j}=I$. It is as yet unknown whether such operators exist for the corresponding Fefferman-Stein decomposition of BMO.

To state the main result of this paper, we need to recall some features of the work of Janson and Uchiyama. First, we shall say that a singular integral operator 
$K$ is homogeneous if it commutes with translations and dilations. This means that, up to a constant multiple of the identity, $K$ can be expressed by

$$
K f(x)=\int_{\mathbb{R}^{n}} \hat{f}(\xi) m(\xi) e^{2 \pi i x \cdot \xi} d \xi
$$

where $m(\xi)$ is homogeneous of degree zero, that is, $m(\xi)=m(\xi /|\xi|)$, and $m(\xi) \in$ $C^{\infty}\left(\mathbb{R}^{n} \backslash 0\right)$.

We shall say that a family $\mathbb{K}=\left(K_{1}, \ldots, K_{M}\right)$ of homogeneous singular integrals satisfies the Janson-Uchiyama condition provided that

$$
\operatorname{rank}\left[\begin{array}{ccc}
m_{1}(\omega) & \ldots & m_{M}(\omega) \\
m_{1}(-\omega) & \ldots & m_{M}(-\omega)
\end{array}\right]=2 \quad\left(\omega \in \Sigma_{n-1}\right) .
$$

Theorem 1.5. Suppose that the family $\mathbb{K}=\left(K_{1}, \ldots, K_{M}\right)$ of homogenous singular integrals satisfies (JUC). Then to each $g \in \mathrm{CMO}\left(\mathbb{R}^{n}\right)$ one can construct a $C Z O$ family $\mathbb{L}=\left(L_{1}, \ldots, L_{M}\right)$ such that $L_{j} g=h_{j} \in B^{2}\left(\mathbb{R}^{n}\right)$ and $g=\sum_{j=1}^{M} K_{j}^{*} h_{j}$. Moreover, $\sum_{j=1}^{M}\left\|h_{j}\right\|_{B^{2}} \leq C\|g\|_{\mathrm{CMO}}$.

Since part of our goal is to make our approach seem as simple as possible, we will begin by making some technical assumptions that, while not critical, do allow us to avoid many technicalities that would only clutter the arguments. First, any singular integral in the sequel will be assumed to map real-valued functions to real-valued functions. Though this hypothesis excludes many important examples, nevertheless Theorem 1.5 continues to hold without it. We will assume that all functions are real-valued as well. Second, Theorem 1.5 will only be proved in the case where one of the operators $K_{1}, \ldots, K_{M}$ in $\mathbb{K}$ is the identity. Again, the arguments are simplified in this case. In any event, neither of these hypotheses exclude the most well-known case, the Fefferman-Stein decomposition.

The proof will rely on some facts about wavelets and singular integrals that we will review first.

\section{Wavelets, Singular INTEgRals, AND CMO}

Here we will review some basic facts about wavelets on $\mathbb{R}^{n}$ and their roles in describing certain Calderón-Zygmund operators and in norming CMO. These facts are either known or follow readily from familiar techniques, so no proofs will be given in this section.

First we recall some very basic properties of orthogonal wavelet bases for $\mathbb{R}^{n}$. We refer to Meyer [5] for more details concerning the construction of wavelets. Let $E=\{0,1\}^{n} \backslash(0,0)$ and let $\mathcal{Q}$ denote the family of dyadic cubes of the form $Q=Q_{j, k}=\left\{x: 2^{j} x-k \in[0,1)^{n}\right\}$. Given $f(x)$ and $Q=Q_{j, k} \in \mathcal{Q}$, we write $f_{Q}(x)=2^{n j / 2} f\left(2^{j} x-k\right)$.

Theorem 2.1 (Existence of wavelets). There is a set $\left\{\psi^{\epsilon}\right\}_{\epsilon \in E}$ of $2^{n}-1$ real-valued 'mother wavelets' such that the functions $\left\{\psi_{Q}^{\epsilon}\right\}_{\epsilon \in E, Q \in \mathcal{Q}}$ form an orthonormal basis for $L^{2}\left(\mathbb{R}^{n}\right)$. Furthermore, the $\psi^{\epsilon}$ can all be chosen to be in $C^{\alpha}\left(\mathbb{R}^{n}\right)$ for any given $\alpha>0$, to have support in $B(0 ; m)$ for some $m>0$, and to have integral zero.

In particular, we will want the wavelets to have continuous derivatives of order at least one. 
Lemma 2.2. Suppose that a homogenous singular integral $L$ and a wavelet $\psi_{Q}^{\epsilon}$ as in Theorem 2.1 with $Q=Q_{j, k}$ having center $x_{Q}$ are given. Then there is a constant $C_{L}$ depending only on $L$ and a $\gamma>0$ such that $\Psi_{Q}^{\epsilon}=L\left(\psi_{Q}^{\epsilon}\right)$ satisfies

$$
\left|\Psi_{Q}^{\epsilon}\right| \leq C_{L} \frac{2^{n j / 2}}{\left(1+2^{j}\left|x-x_{Q}\right|\right)^{n+\gamma}}
$$

and

$$
\left|\Psi_{Q}^{\epsilon}(x)-\Psi_{Q}^{\epsilon}(y)\right| \leq C_{L} 2^{n j / 2} \frac{2^{j}|x-y|}{1+2^{j}|x-y|}\left(\frac{1+2^{j}|x-y|}{1+2^{j}\left|x-x_{Q}\right|}\right)^{n+\gamma} .
$$

The lemma is a standard fact about the images of smooth functions with vanishing integrals under singular integrals. There is nothing special about the wavelets. In fact, we define any function satisfying the conclusions of Lemma 2.2, and having integral zero, to be a vaguelette.

Next, we recall a technical lemma due to Uchiyama [6].

Lemma 2.3. Suppose that $\mathbb{K}=\left(K_{1}, \ldots, K_{M}\right)$ is a system of homogenous singular integrals satisfying (JUC). Then to each $\nu \in \Sigma_{M-1}$ there exists a homogeneous pseudoinverse family $\mathbb{L}_{\nu}=\left(L_{1}, \ldots, L_{M}\right)$ such that

$$
\left\{\begin{array}{l}
\mathbb{K}^{*} \cdot \mathbb{L}_{\nu}=\sum_{l=1}^{M} K_{l}^{*} L_{l}=I, \\
\nu \cdot \mathbb{L}_{\nu}=\sum_{l=1}^{M} \nu_{l} L_{l}=0 .
\end{array}\right.
$$

The constants $C_{L}$ associated with the $\mathbb{L}_{\nu}$ appearing in Lemma 2.2 are bounded independent of $\nu$ by a constant $C_{\mathbb{K}}$.

Such a family $\mathbb{L}_{\nu}$ will be referred to as a $\nu$-pseudoinverse of $\mathbb{K}$. Next, we briefly discuss wavelet-vaguelette operators. These are operators of the form

$$
f \mapsto \sum_{\epsilon \in E, Q \in \mathcal{Q}}\left\langle f, \psi_{Q}^{\epsilon}\right\rangle \Psi_{Q}^{\epsilon}
$$

The $\psi_{Q}^{\epsilon}$ are the wavelets in Theorem 2.1 , but the $\Psi_{Q}^{\epsilon}$ can be any vaguelettes. For the application that we have in mind they will have the form $\vec{\Psi}_{Q}^{\epsilon}=\mathbb{L}_{\nu}\left(\psi_{Q}^{\epsilon}\right)$ where $\nu=\nu_{Q}^{\epsilon}$ will depend on $\epsilon$ and $Q$. Though such operators are no longer homogeneous, they are still nice CZO's:

Proposition 2.5. Any wavelet-vaguelette operator is a Calderón-Zygmund operator. In particular, any operator as in (2.4) is continuous on $L^{2}$, and will map $H A^{1}$ into $A$ and $B^{2}$ into CMO.

The by now standard proof of this fact is left to the reader (cf. [4] and [5], where a precise definition of Calderón-Zygmund operator can also be found).

As a final preparation for the constructive decomposition, we need to make note of the wavelet decomposition of CMO. The proof of the following theorem runs along the same lines as the corresponding result for BMO (see pg. 154 of [5] and Theorem E of [3]).

Theorem 2.6. Suppose that $g \in \mathrm{CMO}$ and wavelets $\psi_{Q}^{\epsilon}$ as in Theorem 2.1 are given. Then $g$ has a wavelet expansion

$$
g \sim \sum_{\epsilon \in E, Q \in \mathcal{Q}} \lambda_{Q}^{\epsilon} \psi_{Q}^{\epsilon}
$$


converging weakly to $g$ and satisfying the central Carleson condition

$$
\left(\sup _{R>0} \frac{1}{R^{n}} \sum_{Q \in \mathcal{Q}, Q \subset B_{2 R}}\left|\lambda_{Q}^{\epsilon}\right|^{2}\right)^{1 / 2} \leq C_{c}\|g\|_{\mathrm{CMO}}
$$

where the supremum is taken over those balls centered at the origin and $C_{c}$ just depends on the wavelet family.

\section{Proof of Theorem 1.5 for $g$ having $\Gamma_{0}$-Type}

In light of Theorem 2.6, we shall say that $g \in \mathrm{CMO}$ has $\Gamma_{0}$-type provided $g=$ $\sum_{\epsilon \in E} \sum_{j=1}^{\infty} \lambda_{j} 2^{n j / 2} \psi^{\epsilon}\left(2^{j} x\right)=\sum_{\epsilon \in E} g^{\epsilon}$. The terminology stems from the fact that the supports of $\psi_{j}^{\epsilon}=2^{n j / 2} \psi^{\epsilon}\left(2^{j} x\right)$, when raised a height $2^{j}$ above $\mathbb{R}^{n}$ inside of $\mathbb{R}_{+}^{n+1}$ all lie inside of a cone centered at the origin. Coupled with Lemma 2.2, this observation lies at the heart of the constructive decomposition in this special case. In the ensuing arguments we shall assume, in addition, that $g=g^{\epsilon}$ for some fixed $\epsilon \in E$. The constructive decomposition of $g$ will then follow by summing the constructive decompositions for $g^{\epsilon}$ over $E$. We shall then write $\psi=\psi^{\epsilon}$ and $g=\sum_{j=1}^{\infty} \lambda_{j} 2^{n j / 2} \psi\left(2^{j} x\right)$. In section 4 we will show how to reduce the constructive decomposition for any $g \in$ CMO to this special case.

Now for each $j=1,2 \ldots$, we will choose below a vector $\nu_{j} \in \Sigma_{M-1}$. Let $\mathbb{L}_{j}=\mathbb{L}_{\nu_{j}}$ be a $\nu_{j}$-pseudoinverse of $\mathbb{K}$ and set $\vec{h}_{j}=\mathbb{L}_{j}\left(\psi_{j}\right)$. Then $\vec{h}_{j}$ satisfies the conclusions of Lemma 2.2 with $x_{Q}=0$. Our goal is to show that $\vec{h}=\sum_{j=1}^{\infty} \lambda_{j} \vec{h}_{j} \in B^{2}$. It clearly suffices to show that the averages $\left\|\vec{h} \chi_{B_{k}}\right\|_{L^{2}} /\left|B_{k}\right|^{1 / 2}$ are uniformly bounded, where $B_{k}=B\left(0 ; 1 / 2^{k}\right)$. This is done by showing the same for $\sum_{j \geq k} \lambda_{j} \vec{h}_{j}$ (Lemma 3.1) and $\sum_{j<k} \lambda_{j} \vec{h}_{j}$ (Lemma 3.3) when the $\vec{h}_{j}$ are chosen appropriately.

Lemma 3.1. For each $k \geq 0$,

$$
\left(\frac{1}{\left|B_{k}\right|} \int_{B_{k}}\left|\sum_{j \geq k} \lambda_{j} h_{j}(x)\right|^{2} d x\right)^{1 / 2} \leq \text { const. }\|g\|_{\mathrm{CMO}}
$$

Proof of Lemma 3.1. $\mathbb{L}=\sum_{j}\left\langle\cdot, \psi_{j}\right\rangle \vec{h}_{j}$ is a vaguelette operator as in (2.4) so, in view of the dependence of $\mathbb{L}$ on $\mathbb{K}$, there is a constant $C_{\mathbb{K}}$ such that the $L^{2}$ operator norm of $\mathbb{L}$ is at most $C_{\mathbb{K}}$, independent of the choices of each $\mathbb{L}_{j}$. Therefore,

$$
\begin{aligned}
\int_{B_{k}}\left|\sum_{j \geq k} \lambda_{j} \vec{h}_{j}(x)\right|^{2} d x & \leq \int_{\mathbb{R}^{n}}\left|\sum_{j \geq k} \lambda_{j} \vec{h}_{j}(x)\right|^{2} d x \\
& \leq C_{\mathbb{K}}^{2} \int_{\mathbb{R}^{n}}\left|\sum_{j \geq k} \lambda_{j} 2^{n j / 2} \psi\left(2^{j} x\right)\right|^{2} d x \\
& =C_{\mathbb{K}}^{2} \sum_{j \geq k}\left|\lambda_{j}\right|^{2} \leq C_{c}^{2} C_{\mathbb{K}^{2}}^{2} 2^{-n k}\|g\|_{\text {CMO }}^{2}=\text { const. }\left|B_{k}\right|\|g\|_{\text {CMO }}^{2}
\end{aligned}
$$

because of orthogonality of the wavelets and the Carleson characterization of CMO. This proves the lemma.

The $\vec{h}_{j}=\mathbb{L}_{j}\left(\psi_{j}\right)$ will be chosen iteratively. For reasons that will become apparent, the $\mathbb{K}$-pseudoinverses $\mathbb{L}_{j}$ will be chosen as $\mathbb{L}_{j}=\mathbb{L}_{\nu_{j}}$ with $\nu_{j}$ proportional to either: (i) $\sum_{1 \leq l<j} \lambda_{l} \vec{h}_{l}(0)$, or (ii) $\lambda_{j} \vec{h}_{j}(0)+2 \sum_{1 \leq l<j} \lambda_{l} \vec{h}_{l}(0)$. In case (i), we will say that $j \in \kappa_{1}$; in case (ii) we shall say that $j \in \kappa_{2}$. While there is no issue as to 
whether $\vec{h}_{j}$ is well-defined in case (i), this does become an issue in case (ii), since $\vec{h}_{j}$ depends first on $\nu_{j}$. This dilemma will be resolved in terms of our criterion below for specifying whether $j \in \kappa_{1}$ or $j \in \kappa_{2}$.

In what follows, let $A$ be a constant such that $A>2 C_{c} C_{\mathbb{K}}$. Notice that by Lemma 2.2 and Theorem 2.6, whenever $|x|<1 / 2^{k}$ and $j \leq k$ one will have

$$
\begin{aligned}
\left|\lambda_{j} \vec{h}_{j}(x)\right| & \leq \frac{A}{2}\|g\|_{\mathrm{CMO}}, \quad \text { and } \\
\left|\lambda_{j}\left(\vec{h}_{j}(x)-\vec{h}_{j}(0)\right)\right| & \leq 2^{j}|x| \frac{A}{2}\|g\|_{\mathrm{CMO}} .
\end{aligned}
$$

Lemma 3.3. The $\vec{h}_{j}$ can be chosen iteratively so that for each $k$,

$$
\frac{1}{\left|B_{k}\right|} \int_{B_{k}}\left|\sum_{j<k} \lambda_{j} \vec{h}_{j}(x)\right|^{2} \leq 5 A^{2}\|g\|_{\mathrm{CMO}}^{2}
$$

To begin the induction, we note that the $k=1$ case is trivial. Next, suppose that $\vec{h}_{1}, \ldots, \vec{h}_{k-1}$ are chosen. If choosing $\nu_{k}$ proportional to $\sum_{j<k} \lambda_{j} \vec{h}_{j}(0)$ allows us to find a pseudoinverse solution $\vec{h}_{k}=\mathbb{L}_{\nu_{k}}\left(\psi_{k}\right)$ such that

$$
\sum_{l \leq k, l \in \kappa_{1}}\left|\lambda_{l} \vec{h}_{l}(x)\right|^{2} \leq 2 A^{2}\|g\|_{\mathrm{CMO}}^{2}
$$

whenever $x \in B_{k}$, then we put $k \in \kappa_{1}$. If it is impossible to choose $\vec{h}_{k}$ this way, then any pseudoinverse solution $\vec{h}_{k}$ with $\nu_{k}$ proportional to $\sum_{j<k} \lambda_{j} \vec{h}_{j}(0)$ must satisfy

$$
\left|\lambda_{k} \vec{h}_{k}(x)\right|^{2}+\sum_{l<k, l \in \kappa_{1}}\left|\lambda_{l} \vec{h}_{l}(x)\right|^{2}>2 A^{2}\|g\|_{\mathrm{CMO}}^{2}
$$

for some $x \in B_{k}$. We then put $k \in \kappa_{2}$, though we must show the well-definedness of $\nu_{k}$ in that case.

Lemma 3.6. If (3.5) is necessitated for some $x \in B_{k}$, then one can find a pseudoinverse solution $\vec{h}_{k}=\mathbb{L}_{k} \psi_{k}$, such that $\left\langle\vec{h}_{k}(x), \vec{h}_{k}(0)+2 \sum_{j<k} \vec{h}_{j}(0)\right\rangle_{\mathbb{R}^{M}} \equiv 0$.

Proof of Lemma 3.6. In view of (3.2), any choice of $\vec{h}_{k}=\mathbb{L}_{\nu}\left(\psi_{k}\right)$ will satisfy $\left|\lambda_{k} \vec{h}_{k}(x)\right| \leq \frac{1}{2} A\|g\|_{\mathrm{CMO}}$ independent of $x$. Thus, (3.5) implies that

$$
\sum_{j<k, j \in \kappa_{1}}\left|\lambda_{j} \vec{h}_{j}\left(x_{0}\right)\right|^{2}>\frac{7}{4} A^{2}\|g\|_{\mathrm{CMO}}^{2}
$$

for some $x_{0} \in B_{k}$.

Next, observe that

$$
\begin{aligned}
\left|\sum_{j \leq k-1} \lambda_{j} \vec{h}_{j}(0)\right|^{2} & =\sum_{j \leq k-1}\left\langle\lambda_{j} \vec{h}_{j}(0), \lambda_{j} \vec{h}_{j}(0)+2 \sum_{l<j} \lambda_{l} \vec{h}_{l}(0)\right\rangle \\
& =\sum_{j<k, j \in \kappa_{1}}\left|\lambda_{j} \vec{h}_{j}(0)\right|^{2}
\end{aligned}
$$


because of the definition of $\kappa_{i}$ and the induction hypothesis. Since $x_{0} \in B_{k}$,

$$
\begin{aligned}
& \sum_{j<k, j \in \kappa_{1}}\left|\lambda_{j} \vec{h}_{j}(0)\right|^{2}=\sum_{j<k, j \in \kappa_{1}}\left[\left|\lambda_{j} \vec{h}_{j}(0)\right|^{2}-\left|\lambda_{j} \vec{h}_{j}\left(x_{0}\right)\right|^{2}\right]+\left|\lambda_{j} \vec{h}_{j}\left(x_{0}\right)\right|^{2} \\
& \quad=\sum_{j<k, j \in \kappa_{1}} \lambda_{j}^{2}\left[\left\langle\vec{h}_{j}(0), \vec{h}_{j}(0)-\vec{h}_{j}\left(x_{0}\right)\right\rangle+\left\langle\vec{h}_{j}(0)-\vec{h}_{j}\left(x_{0}\right), \vec{h}_{j}\left(x_{0}\right)\right\rangle\right]+\left|\lambda_{j} \vec{h}_{j}\left(x_{0}\right)\right|^{2} \\
& \geq \frac{7}{4} A^{2}\|g\|_{\mathrm{CMO}}^{2}-\frac{1}{2}\left(\sum_{j \leq k-1} 2^{j}\left|x_{0}\right|\right) A^{2}\|g\|_{\mathrm{CMO}}^{2} \\
& \quad>\frac{5}{4} A^{2}\|g\|_{\mathrm{CMO}}^{2}
\end{aligned}
$$

because (3.2) applies uniformly to any choice of $\vec{h}_{k}$. This shows that for $\vec{H}=$ $\sum_{j<k} \lambda_{j} \vec{h}_{j}(0)$, one has $|\vec{H}|^{2}>\frac{5}{4} A^{2}\|g\|_{\mathrm{CMO}}^{2}$. This is just what we need to solve for $\vec{h}_{k}$.

Let $K \subset \mathbb{R}^{M}$ be the closed convex hull of those unit vectors whose angles with $\vec{H}$ are at most $\arccos (3 / 5)$. By (3.2), for each $\nu \in \Sigma_{M-1}$ we can find $\vec{h}_{k, \nu}$ such that $\left|\lambda_{k} \vec{h}_{k, \nu}(x)\right|^{2} \leq \frac{1}{4} A^{2}\|g\|_{\text {CMO }}^{2}<\frac{1}{5}|\vec{H}|^{2}$. The solution $\vec{h}_{k, \nu}$ can be chosen to vary smoothly with $\nu$ (this is due to Uchiyama's observations). Consider the mapping

$$
\Theta: \nu \mapsto \frac{\lambda_{k} \vec{h}_{k, \nu}(0)+2 \vec{H}}{\left|\lambda_{k} \vec{h}_{k, \nu}(0)+2 \vec{H}\right|}
$$

The mapping $\Theta(\nu)$ can be extended to $K$ by setting $\Theta(\mu)=\Theta\left(\frac{\mu}{|\mu|}\right)$ (note that $0 \notin$ $K)$. But then $\Theta$ sends $K$ into itself. This means that if $\nu \in K$, then $(\Theta(\nu) \cdot \vec{H} /|\vec{H}|)>$ $3 / 5$, which follows from the observation that $\left|\lambda_{k} \vec{h}_{k, \nu}(0)\right|<\frac{1}{2}|\vec{H}|$. Therefore $\Theta$ is a continuous mapping from the compact, convex set $K$ into itself. Brouwer's fixed point theorem then guarantees that $\Theta$ has a fixed point in $\Sigma_{M-1}$. The lemma now follows from the definition of $\Theta$.

Remark. Since we are after a constructive decomposition, every step must be constructive. Brouwer's fixed point theorem is only an existence result. But, in this particular case, the fixed point can be constructed by optimization techniques since $\Theta$ and its derivatives can be computed explicitly. In fact, similar techniques must be used to verify (3.4) or (3.5) to begin with. It is not obvious that the definitions of $\kappa_{1}, \kappa_{2}$ are constructive. They can be made so by fixing a canonical choice of $\mathbb{L}_{\nu}$ in (2.3) for each $\nu \in \Sigma_{M-1}$ (cf. [6]). If (3.4) then fails for the 'canonical' $\vec{h}_{k}-$ which is decidable by similar optimization techniques - the same estimate for $|\vec{H}|$, and therefore Lemma 2.6, still hold.

Proof of Lemma 3.3. It just remains to show that

$$
\int_{B_{k}}\left|\sum_{j<k} \lambda_{j} \vec{h}_{j}(x)\right|^{2} d x \leq \text { const. }\left|B_{k}\right|\|g\|_{\mathrm{CMO}}
$$


with constant independent of $k$. Now fix $k$. Then by the definitions of $\kappa_{1}$ and $\kappa_{2}$ and by Lemma 3.6,

$$
\begin{aligned}
\left|\sum_{j<k} \lambda_{j} \vec{h}_{j}(x)\right|^{2} & =\sum_{j \in \kappa_{1}, j<k}\left[\left|\lambda_{j} \vec{h}_{j}(x)\right|^{2}+2 \sum_{l<j} \lambda_{j} \lambda_{l}\left\langle\vec{h}_{l}(x)-\vec{h}_{l}(0), \vec{h}_{j}(x)\right\rangle\right] \\
& +\sum_{j \in \kappa_{2}, j<k}\left[\lambda_{j}^{2}\left\langle\vec{h}_{j}(x), \vec{h}_{j}(x)-\vec{h}_{j}(0)\right\rangle+2 \sum_{l<j} \lambda_{j} \lambda_{l}\left\langle\vec{h}_{j}(x), \vec{h}_{l}(x)-\vec{h}_{l}(0)\right\rangle\right] \\
& =I+I I .
\end{aligned}
$$

By (3.4), if $x \in B_{k}$, then

$$
\sum_{j \in \kappa_{1}}\left|\lambda_{j} \vec{h}_{j}(x)\right|^{2}<2 A^{2}\|g\|_{\text {CMO }}^{2}
$$

which gives a desired bound for the first term in $I$.

For the other terms we apply (3.2) to estimate

$$
\left|\lambda_{j} \lambda_{l}\left\langle\vec{h}_{j}(x), \vec{h}_{l}(x)-\vec{h}_{l}(0)\right\rangle\right| \leq 2^{l}|x| \frac{1}{4} A^{2}\|g\|_{\mathrm{CMO}}^{2}
$$

whenever $l \leq j<k$ and $x \in B_{k}$. Therefore

$$
\sum_{l \leq j<k}\left|\lambda_{j} \lambda_{l}\left\langle\vec{h}_{j}(x), \vec{h}_{l}(x)-\vec{h}_{l}(0)\right\rangle\right| \leq \frac{1}{4} A^{2}\|g\|_{\mathrm{CMO}}^{2} \sum_{l \leq j<k} 2^{l} \leq \frac{2^{k}|x|}{2} A^{2}\|g\|_{\mathrm{CMO}}^{2}
$$

This gives desired bounds for the second terms in $I$ and $I I$. Similar observations show that the first term in $I I$ is bounded by $\frac{1}{4} 2^{k}|x| A^{2}\|g\|_{\text {CMO }}^{2}$ when $x \in B_{k}$. The proof of Lemma 3.3 is then completed by taking the average of these inequalities over $B_{k}$. The constant $5 A$ can be improved somewhat.

Lemmas 3.1 and 3.3 together show that, for appropriate choices of $\vec{h}_{j}=\mathbb{L}_{j} \psi_{j}$, one gets $\vec{h}=\sum_{j=0}^{\infty} \lambda_{j} \vec{h}_{j} \in B^{2}$ with $\|\vec{h}\|_{B^{2}} \leq 2 A\|g\|_{\text {CMO }}$. Since $\mathbb{L}=\sum_{j=0}^{\infty}\left\langle\cdot, \psi_{j}\right\rangle \vec{h}_{j}$ is a vaguelette operator of the desired form, this proves Theorem 1.5 in the case where $g \in \mathrm{CMO}$ is of $\Gamma_{0}$-type.

\section{Reduction to $\Gamma_{0}$-Type}

Before proceeding further we make a few additional comments along the lines of Uchiyama's work. First, just as Uchiyama did, we can assume for convenience that $g$ has compact support. In this case one can show that in the wavelet expansion of $g$, $\lim _{k \rightarrow \infty}\left\|\sum_{Q: l(Q)>2^{k}} \lambda_{Q}^{\epsilon} \psi_{Q}^{\epsilon}\right\|_{\mathrm{CMO}}=0$ (cf. Lemma 3.4 of [2]). By an approximation and rescaling argument, then, to prove Theorem 1.5 it suffices to assume that $g=\sum_{Q: l(Q)<1} \lambda_{Q}^{\epsilon} \psi_{Q}^{\epsilon}$.

With such assumptions on $g \in \mathrm{CMO}$, we write $g \sim \sum_{\epsilon \in E, Q \in \mathcal{Q}} \lambda_{Q}^{\epsilon} \psi_{Q}^{\epsilon}$ as $g=g_{1}+$ $g_{2}$. Here, under the assumption that each $\psi_{Q}$ is supported in $B\left(x_{Q}, m l(Q)\right), g_{1} \sim$ $\sum_{\epsilon \in E, Q \in \mathcal{Q}^{\prime}} \lambda_{Q}^{\epsilon} \psi_{Q}^{\epsilon}$ where $\mathcal{Q}^{\prime}$ is the set of those cubes such that $0 \in B\left(x_{Q}, 3 m l(Q)\right)$. Thus the supports of the wavelets associated to $\mathcal{Q}^{\prime}$ have length proportional to the distance from $x_{Q}$ to the origin. $g_{2}$ is the sum over the remaining wavelet terms. When written this way, one sees that for each level $j$, there are at most a fixed finite number of cubes $Q \in \mathcal{Q}^{\prime} \cap \mathcal{Q}_{j}$ where $\mathcal{Q}_{j}=\left\{Q \in \mathcal{Q}: l(Q)=1 / 2^{j}\right\}$.

It turns out that $g_{2} \in B^{2}$ already. That is:

Lemma 4.1. In the decomposition $g=g_{1}+g_{2}$ above, one has $g_{2} \in B^{2}$ with $\left\|g_{2}\right\|_{B^{2}} \leq C\|g\|_{\mathrm{CMO}}$ where $C$ just depends on the particular choice of wavelet basis. 
Proof of Lemma 4.1. Let $B$ be any ball centered at the origin. Rewrite $g_{2}=g_{2}^{i}+$ $g_{2}^{e}+g_{2}^{o}$ where the wavelets occurring in $g_{2}^{i}$ are supported completely inside of $B$ and those occurring in $g_{2}^{o}$ are supported completely outside of $B$. It is clear, then, that

$$
\frac{1}{|B|} \int_{B}\left|g_{2}\right|^{2}=\frac{1}{|B|} \int_{B}\left|g_{2}^{i}+g_{2}^{e}\right|^{2}=\frac{1}{|B|} \int_{B}\left|g_{2}^{i}\right|^{2}+\left|g_{2}^{e}\right|^{2}
$$

because the wavelets occurring in $g_{2}^{i}$ are supported inside of $B$ and are orthogonal to those occurring in $g_{2}^{e}$. By the definition of $g_{2}$, any wavelet occurring in $g_{2}^{e}$ must be completely supported in $2 B$. Thus the right-hand side is clearly bounded by

$$
\frac{1}{|B|} \int_{2 B}\left|g_{2}^{i}\right|^{2}+\left|g_{2}^{e}\right|^{2} \leq \frac{1}{|B|} \sum_{Q \subset 2 B}\left|\lambda_{Q}^{\epsilon}\right|^{2} \leq \text { const. }\|g\|_{\mathrm{CMO}}^{2}
$$

The proof is completed by noting that $B$ is any ball centered at the origin.

We now complete the reduction to the $\Gamma_{0}$-type case under the hypothesis that the family $\mathbb{K}$ in Theorem 1.5 includes the identity operator. First note that $g_{1}$ can be split into a finite sum of terms each of which has the form

$$
g_{1}^{r}=\sum_{\epsilon \in E} \sum_{j \in \mathbb{Z}} \lambda_{j, r}^{\epsilon} 2^{n j / 2} \psi^{\epsilon}\left(2^{j} x-r\right) .
$$

The $g_{1}^{r}$ are indexed by $r \in \mathbb{Z}^{n} \cap B(0,3 m)$ : this is essentially the condition that the center of $Q=Q_{j, r}$ has distance at most $3 m$ from the origin. In view of Lemma 2.2 , the argument applied to $g$ having $\Gamma_{0}$-type in section 3 works equally well for each $g_{1}^{r}$ to show that there is an $\vec{h}_{1}^{r} \in \vec{B}^{2}$ with $\mathbb{K}^{*} \vec{h}_{1}^{r}=g_{1}^{r}$. Since there are at most $\#\left(\mathbb{Z}^{n} \cap B(0,3 m)\right)$ such $r$, this shows that $g_{1}$ has the desired constructive decomposition.

On the other hand, if $I$ is one of the operators occurring in $\mathbb{K}, I=K_{1}$, say, then by Lemma $4.1, \vec{h}_{2}=\left(g_{2}, 0, \ldots, 0\right)$ is itself an element of $B^{2}$ satisfying $\mathbb{K}^{*} \vec{h}_{2}=g_{2}$. This proves the constructive decomposition Theorem 1.5 in the case where $I$ occurs in $\mathbb{K}$.

Remark. To prove the general case of Theorem 1.5, that is, when the identity does not necessarily occur on $\mathbb{K}$, one needs to perform a constructive decomposition on $g_{2}$. It is not true that any singular integral will map $g_{2}$ into $B^{2}$, though it will map $g_{2}$ into CMO, of course. As above, it is not difficult to reduce everything to establishing an analogue of Lemma 3.6. Let $g_{2, j}$ be the sum of wavelet terms in $g_{2}$ at scale $j$. Then one wishes to choose pseudo-inverse operators such that $\vec{h}_{2, j}=\mathbb{L}_{2, j} g_{2, j}$ will

be orthogonal at each point either to $\sum_{l<j} \vec{h}_{2, l}(0)$ or to $\vec{h}_{2, j}(0)+2 \sum_{l<j} \vec{h}_{2, l}(0)$ according to a criterion similar to that used in the $\Gamma_{0}$-type case. This is possible, but the estimates are more technical in the general case.

\section{ACKNOWLEDGEMENTS}

The author would like to thank John Gilbert for introducing him to the spaces $H A^{p}$, Caroline Sweezy for some comments leading to the proof of Theorem 1.5, and the referee for several helpful suggestions. 


\section{REFERENCES}

1. J.-A. Chao, J.E. Gilbert and P.A. Tomas, Molecular decompositions in $H^{p}$-theory, Supplement Rend. Circ. Mat. Palermo (1981), 115-119. MR 83c:42016

2. M. Christ and D. Geller, Singular integral characterizations of Hardy spaces on homogeneous groups, Duke Math. J. 51 (1984), 547-598. MR 86g:43007a

3. Y.-Z. Chen and K.-S. Lau, Some new classes of Hardy spaces, J. Functional Anal. 84 (1989), 255-278. MR 90f:46059

4. J. García-Cuerva, Hardy spaces and Beurling algebras, J. London Math. Soc. 39 (1989), 499513. MR 90i: 42032

5. Y. Meyer, Ondelettes et operateurs. I, II, III, Hermann ed. Paris (1990). MR 93i:42002; MR 93i:42003; MR 93i: 42004

6. A. Uchiyama, A constructive proof of the Fefferman-Stein decomposition of $\mathrm{BMO}\left(\mathbf{R}^{n}\right)$, Acta Math. 148 (1982), 215-241. MR 84h:42037

7. T. Wolff, Counterexamples with harmonic gradients in $\mathbf{R}^{3}$, in "Essays on Fourier Analysis in Honor of Elias M. Stein," 321-384, Princeton University Press, Princeton, N.J., 1995. MR 95m:31010

Department of Mathematical Sciences, New Mexico State University, Las Cruces, New Mexico 88003-8001

E-mail address: jlakey@nmsu.edu 